University of Texas Rio Grande Valley

ScholarWorks @ UTRGV

Psychological Science Faculty Publications and

Presentations

College of Liberal Arts

$6-2020$

\title{
Lifetime Interpersonal Victimization Profiles and Mental Health Problems in a Nationally Representative Panel of Trauma- Exposed Adults from the United Kingdom
}

\author{
Ruby Charak \\ The University of Texas Rio Grande Valley, ruby.charak@utrgv.edu \\ Maria Louison Vang \\ Ulster University \\ Mark Shevlin \\ Ulster University \\ Menachem Ben-Ezra \\ Ariel University \\ Thanos Karatzias \\ Napier University
}

See next page for additional authors

Follow this and additional works at: https://scholarworks.utrgv.edu/psy_fac

Part of the Psychology Commons

\section{Recommended Citation}

Charak, R., Vang, M. L., Shevlin, M., Ben-Ezra, M., Karatzias, T., \& Hyland, P. (2020). Lifetime Interpersonal Victimization Profiles and Mental Health Problems in a Nationally Representative Panel of TraumaExposed Adults from the United Kingdom. Journal of Traumatic Stress. https://doi.org/10.1002/jts.22527

This Article is brought to you for free and open access by the College of Liberal Arts at ScholarWorks @ UTRGV. It has been accepted for inclusion in Psychological Science Faculty Publications and Presentations by an authorized administrator of ScholarWorks @ UTRGV. For more information, please contact justin.white@utrgv.edu, william.flores01@utrgv.edu. 


\section{Authors}

Ruby Charak, Maria Louison Vang, Mark Shevlin, Menachem Ben-Ezra, Thanos Karatzias, and Philip Hyland 
Lifetime Interpersonal Victimization Profiles and Mental Health Problems in a Nationally Representative Panel of Trauma-Exposed Adults from the United Kingdom

Ruby Charak1, Maria Louison Vang2, Mark Shevlin3, Menachem Ben-Ezra4, Thanos Karatzias5, and Philip Hyland6

1Department of Psychological Science, The University of Texas Rio Grande Valley, Edinburg, Texas, USA ${ }_{2}$ School of Psychology, Ulster University, Coleraine, Northern Ireland 3School of Psychology and Psychology Research Institute, Ulster University, Coleraine, Northern Ireland 4School of Social-Work, Ariel University, Ariel, Israel 5Edinburgh Napier University, School of Health and Social Care, Edinburgh, UK 6Department of Psychology, Maynooth University, Ireland

\section{Author Note}

Ruby Charak received funding for this work from the Office of Faculty Affairs and Diversity, The University of Texas Rio Grande Valley, Edinburg, Texas.

Correspondence concerning this article should be addressed to Ruby Charak, Assistant Professor, Department of Psychological Science, The University of Texas Rio Grande Valley, EIEAB 3.207, 1201 W. University Dr., Edinburg, Texas 78539. E-mail: charakruby@gmail.com

\section{APA citation.}

Charak R., Vang, M. L., Shevlin, M., Ben-Ezra, M., Karatzias, T., \& Hyland, P. (2020). Lifetime interpersonal victimization profiles and mental health problems in a nationally representative panel of trauma-exposed adults from the United Kingdom. Journal of Traumatic Stress Studies. Advance online publication. doi.10.1002/jts.22527. 


\begin{abstract}
Exposure to traumatic events has been associated with negative psychological outcomes. There is, however, a dearth of research on revictimization. The current study examined patterns of lifetime interpersonal victimization, based on six types of childhood maltreatment, physical and sexual assault, and assault with a weapon during adulthood via latent class analysis (LCA), with gender as covariate. Using a three-step approach, we assessed differences across the latent classes in symptoms and diagnosis of depression, anxiety, and DSM-5 posttraumatic stress disorder. An adult sample representative of the United Kingdom population with exposure to trauma $(N=1,051)$ was recruited online through a research panel. The mean participant age 47.18 years ( $S D=15.00$, range: $18-90$ years; $68.4 \%$ female). The LCA identified five classes: lifetime polyvictimization $(8.3 \%$; $69.5 \%$ female), sexual revictimization $(13.7 \% ; 96.5 \%$ female), physical revictimization $(12.5 \%$; $1.5 \%$ male), childhood trauma $(25.9 \% ; 85.6 \%$ female), and limited victimization $(39.7 \%$; $40.3 \%$ female). Compared to the other classes, the polyvictimization class followed by the childhood trauma class, demonstrated the highest scores on anxiety, depression, and posttraumatic stress symptoms. The polyvictimization class had nearly a nine- to 33-fold increase in risk of depression (OR (95\% CI): 9.48 (3.34 - 26.87)), anxiety (OR (95\% CI): 12.10 (5.36 - 27.36)), and PTSD diagnoses (OR (95\% CI): 33.63 (16.35 -69.43)) compared to the limited victimization class. The findings facilitate the identification of individuals at risk for revictimization and indicate that evidence-based clinical interventions should be targeted toward those with exposure to revictimization and childhood trauma exposure to alleviate mental health challenges.
\end{abstract}


Lifetime Interpersonal Victimization Profiles and Mental Health Problems in a Nationally Representative Panel of Trauma-Exposed Adults from the United Kingdom Interpersonal victimization, defined as violence perpetrated on one individual by another, is pervasive across developmental periods during the lifetime and includes experiences such as childhood abuse and neglect and adult sexual and physical assault (Charak, Ford, Modrowski, \& Kerig, 2019; Lagdon, Armour, \& Stringer, 2014; Rivera, Fincham, \& Bray, 2018). Exposure to lifetime interpersonal victimization can result in myriad negative psychological consequences, such as posttraumatic stress disorder (PTSD), personality pathology, depression, anxiety, and suicidal behavior (Ford, Charak, Modrowski, \& Kerig, 2018; McElroy et al., 2016). Prior studies have indicated a robust association between experiences of childhood maltreatment and adolescent and/or adulthood victimization whereby exposure to childhood maltreatment increases the risk of victimization during the succeeding developmental stages (i.e., adolescence and adulthood) — a phenomenon referred to as "revictimization" (Charak, DiLillo, MessmanMoore, \& Gratz, 2018; Messman-Moore \& Long, 2003). However, most studies that have examined patterns of lifetime interpersonal victimization and revictimization have used North American samples (Houston, Shevlin, Adamson, \& Murphy, 2011). Thus, to develop effective, generalizable strategies for prevention and intervention, there is a need to examine the impact of lifetime victimization in other regions of the world. With this in mind, the present study aimed to investigate patterns of childhood and adulthood interpersonal victimization and examine their associations with psychological problems in a nationally representative panel of trauma-exposed adults from the United Kingdom.

A popular theory that aids in our understanding of the revictimization phenomenon is the "traumagenic dynamics" model (Finkelhor \& Browne, 1985). In particular, the model suggests that the negative outcomes of childhood sexual abuse (CSA), such as traumatic sexualization (e.g., rewarding a child for sexual activity), may be associated with an increase in risky sexual 
behavior and that experiencing powerlessness may lead to impaired coping strategies, which, in turn, can lead to revictimization. Based on the traumagenic dynamics model, Messman-Moore and Long (2003) suggested that the risk for revictimization is increased among CSA survivors as a result of two mechanisms: (a) engaging in higher rates of risky behavior, such as risky sexual activity, and (b) psychological vulnerability as a result of traumatic stress symptoms, including domains related to PTSD, dissociation, depression, and other impairments. In support of this, the results of several longitudinal studies have shown that symptoms of depression (Culatta, ClayWarner, Boyle, \& Oshri, 2017; Miron \& Orcutt, 2014), anxiety, and posttraumatic stress (Ullman, Najdowski, \& Filipas, 2009) mediate the association between CSA and sexual assault during adulthood.

A second theoretical framework that informs the psychological sequelae of revictimization is the "cumulative trauma" theory, which postulates that as exposure to different types of trauma and victimization increases (e.g., revictimization), these experiences accumulate to exert an additive and detrimental effect on mental health problems. For example, researchers found that among a community sample of men, participants with revictimization experiences exhibited higher levels of anger and more alcohol and drug use compared to those who had been identified as having being victimized during a single developmental stage (e.g., childhood maltreatment; Charak, Eshelman, \& Messman-Moore, 2019). Together, the two theoretical frameworks described suggest that psychological vulnerabilities, such as symptoms of depression, anxiety, and posttraumatic stress that result from childhood trauma exposure, can lead to sexual revictimization experiences, which, in turn, can result in elevated symptoms of mental health problems (Filipas \& Ullman, 2006; Kimerling, Alvarez, Pavao, Kaminski, \& Baumrind, 2007; Walsh et al., 2012).

In addition, it is relevant to note that the literature on revictimization mostly focuses on the homotypic continuity between CSA and adult sexual assault (e.g., Charak et al., 2018; 
Messman-Moore \& Long, 2003) despite a demonstration of heterotypic continuity wherein CSA is associated with adult physical and sexual assault (Kimerling et al., 2007). For instance, the findings from a population-based study of 11,056 women from the United States indicated that CSA exposure led to adult physical and sexual assault, although the association with adult sexual assault was significantly stronger than the association with adult physical assault (Kimerling et al., 2007). Notably, these studies examined the role of one specific child maltreatment type, such as CSA, despite a plethora of work indicating that different types of childhood maltreatment often co-occur and have a cumulative negative effect on the survivor's mental health, which can include depression, posttraumatic stress symptoms, anxiety, and suicidal behavior (Charak, Koot, Dvorak, Elklit, \& Elhai, 2015; Charak et al., 2016; McLafferty et al., 2015; Rivera et al., 2018). Additional research has demonstrated that exposure to multiple types of childhood maltreatment increases the risk for revictimization during adulthood (Charak et al., 2019a; Classen, Palesh, \& Aggarwal, 2005; Widom, Czaja, \& Dutton, 2008). For example, in a large sample of female college students, researchers found that those who reported exposure to two or more types of childhood maltreatment were three times more likely to be sexually revictimized compared to women who had exposure to one or no childhood maltreatment (Jankowski, Leitenberg, Henning, \& Coffey, 2002). However, to our knowledge, no study to date has simultaneously examined patterns of lifetime victimization based on exposure to diverse childhood maltreatment types and different types of adulthood victimization, along with their differential effects on psychological problems. The present study aimed to bridge this gap in the literature to facilitate the development of effective procedures for responding to reports of victimization and revictimization and related psychological sequelae.

The past decade has witnessed a surge in literature using advanced statistical analysis, such as latent class analysis (LCA), to examine the cumulative effect of exposure to multiple types of victimization (e.g., Armour \& Sleath, 2014; Charak \& Koot, 2015). Latent class analysis 
is a person-centered statistical approach (Nylund, Asparouhov, \& Muthén, 2007) that focuses on the identification of subgroups of individuals with similar victimization profiles. Separately, researchers have recommended the use of LCA when investigating co-occurring types of child maltreatment (Rivera et al., 2018) as well as different characteristics of victimization or types of interpersonal victimization that occur in adults (Charak et al., 2018). For the present study, LCA is of further applicability given that additional studies have indicated differences in the distribution of men and women across latent classes of childhood maltreatment and trauma exposure (Ballard et al., 2015; Charak et al., 2018), with the results of some national surveys suggesting differences in prevalence of interpersonal victimization among men and women (Black et al., 2010). For instance, Ballard et al. (2015) found that, based on nine types of childhood traumatic experiences, three latent classes emerged wherein a class characterized by sexual assault comprised primarily female participants, a class with violence exposure comprised primarily male participants, and a class characterized by low levels of childhood trauma exposure had an equal distribution of male and female participants (Ballard et al., 2015). Similarly, findings from the U.S.-based National Intimate Partner and Sexual Violence Survey indicated that women reported higher rates of sexual and physical victimization compared to men (Black et al., 2010).

Furthermore, studies that have used LCA have indicated heterogeneity in patterns of exposure to childhood maltreatment and interpersonal victimization during adulthood (see Contractor, Caldas, Fletcher, Shea, \& Armour, 2018; O'Donnell et al., 2017). Of note, most of the study samples were limited to women except for a study by Houston et al. (2011), in which four latent classes based on lifetime traumatic experiences were identified in a nationally representative sample of adults from the United States; these classes were categorized as high risk, exposure to nonsexual adult interpersonal/noninterpersonal trauma, intermediate risk/sexual abuse, and low risk. Furthermore, the authors found that male participants were more likely to be 
in the class categorized by exposure to nonsexual adult interpersonal/noninterpersonal trauma, and female participants were more likely to be in the intermediate risk/sexual abuse class (Houston et al., 2011). Cognizant of gender differences in exposure to lifetime victimization, in the present study, we additionally controlled for the effect of gender when examining patterns of victimization across the lifespan.

Thus, the current study employed LCA to identify patterns of lifetime victimization in a nationally representative panel of trauma-exposed adults from the United Kingdom. Based on prior studies (Messman-Moore \& Long, 2003; O'Donnell et al., 2017; Widom et al., 2008), we hypothesized that there would be varying patterns (i.e., latent classes) of lifetime interpersonal victimization, with at least one class categorized by exposure to interpersonal victimization during the developmental stages of childhood and adulthood - the "revictimization" class—after controlling for the effect of gender. Drawing from the psychological vulnerability hypothesis of the traumagenic dynamics model and in accordance with the cumulative trauma framework, we additionally hypothesized that participants with membership in the latent classes characterized by revictimization experiences would score higher on measures related to symptoms of depression, anxiety, and posttraumatic stress as compared to those in other classes characterized by victimization experiences that occurred during a single developmental phase or minimal victimization across the lifespan.

\section{Method}

\section{Participants and Procedure}

An adult sample of the United Kingdom population was drawn from an existing online research panel representative of the entire national adult population. An aggregated panel of respondents whose GEO-IP address (i.e., locating a computers' geographic location by identifying the terminal's IP address) was based in the United Kingdom were randomly recruited through probability-based sampling with an aim of recruiting approximately 1,000 participants. 
Invitations were sent out in waves, and the response rate was monitored in the field. Respondents went through prescreening criteria check in which they were asked about their age and gender. This was done to gain a nationally representative sample based on two inclusion criteria: (a) the participant was born in the United Kingdom and (b) the participant was 18 years of age or older at the time of the survey. If these criteria were met, a third inclusion criterion-screening positive for at least one traumatic event exposure in their lifetime-was assessed, using the Life Events Checklist (LEC; Weathers, Blake, Schnurr, Kaloupek, Marx, \& Keane, 2013).

Participants who endorsed any item on the LEC then completed the remaining battery of survey measures. In total 2,653 panel members were contacted, and 1,051 individuals qualified for inclusion in the final analyses (39.6\% selection rate). The National College of Ireland granted the ethical approval for the present study. No incentives were offered for participation. Informed consent was taken at the start of the survey.

The mean participant age 47.18 years $(S D=15.00$, range: $18-90$ years), and $68.4 \%(n=$ 719) of participants were female. The majority of individuals indicated that they were in a committed relationship $(70.4 \%, n=740)$, did not have children under 16 years of age $(67.5 \%, n$ $=709)$, had completed a college or university education $(62.7 \%, n=659)$, and were employed in a full- or part-time position $(58.5 \%, n=615)$. A number of participants indicated that they had emigrated at some point in their lifetime $(17.8 \%, n=187)$.

\section{Measures}

Interpersonal victimization. Participants were asked to provide information on childhood and adulthood victimization using the Adverse Childhood Experiences questionnaire (ACE; Felitti et al., 1998) and a modified version of the LEC (Weathers, Blake, et al., 2013) for the fifth edition of the Diagnostic and Statistical Manual of Mental Disorders (DSM-5). The ACE is a 10-item self-report measure that assesses three types of childhood adversity: abuse, neglect, and household dysfunction. The modified LEC-5 is a 17-item, self-report screening tool 
for lifetime exposure to 16 potentially traumatizing events; the LEC also includes one openended question that allows respondents to describe any other traumatic event not listed (Weathers, Blake, et al., 2013). The modified version of the measure asked participants to respond yes or no to each item to indicate if it happened in childhood (i.e., before age 18) or happened in adulthood (i.e., at or after age 18).

Six types of childhood trauma were assessed using items from both the ACE and LEC. The ACE was used to assess neglect (two items: no one loved me/made me feel special; didn't have enough to eat/had to wear dirty clothes/no one to protect me), emotional abuse (one item: parent swear/insult/humiliate you/afraid that you may be physically hurt), and witnessing interpersonal violence (one item: kick/bite/hit each other/threaten with a weapon), and the LEC was used to assess sexual victimization (two items: rape/attempted rape/sexual acts through force or threat to harm, other unwanted/uncomfortable sexual experience), physical assault (one item: being attacked/hit/slapped/kicked), and assault with a weapon (one item; being shot/stabbed/threatened with weapon) that occurred before the age of 18 years.

Three types of adult victimization were assessed using the LEC: exposure to sexual victimization (two items), physical assault (one item), and assault with a weapon (one item) after the age of 18 years. All variables were dichotomized, with a type of victimization regarded as present if respondents endorsed a minimum of one item within the category.

Depression. The Patient Health Questionnaire-9 (PHQ-9; Kroenke, Spitzer, \& Williams, 2001) was used to assess symptoms of depression. The PHQ-9 is a nine-item self-report measure scored on a 4-point Likert scale ranging from 0 (not at all) to 3 (nearly every day). The scale was developed as part of the Primary Care Evaluation of Mental Health Disorders (PRIME-MD) initiative to diagnose depression in primary health care settings. Higher scores indicate a higher symptom severity, with a cutoff score of 10 indicating moderate levels of depressive symptomatology. Total scores range from 0 to 27 . The internal reliability of the scale has been 
found to be high in prior studies (Cronbach's $\alpha=.89$; Kroenke et al., 2001) and was similarly high in the current study, Cronbach's $\alpha=.94$.

Anxiety. The Generalized Anxiety Disorder-7 (GAD-7; Spitzer, Kroenke, Williams, \& Löwe, 2006), which was developed as part of the PRIME-MD initiative to diagnose anxiety in a primary health care setting, was used to assess symptoms of generalized anxiety. The GAD-7 is a seven-item, self-report measure scored the same way as the PHQ-9 (i.e., on a scale of 0 to 3). Higher total scores indicate a higher severity of anxiety symptoms, with cutoff scores of 10 and 15 indicating moderate and severe levels of anxiety, respectively. Total scores on the GAD-7 range from 0 to 21 . The scale has demonstrated high levels of internal reliability in prior studies (Cronbach's $\alpha=.92$; Spitzer et al., 2006); this was maintained in the current study, Cronbach's $\alpha$ $=.95$.

Posttraumatic stress disorder. The PTSD-Checklist for DSM-5-Civilian Version (PCL5; Weathers, Litz, et al., 2013) was used to assess PTSD symptoms associated with the most distressing trauma reported on the LEC-5. The PCL-5 is a 20-item, self-report measure that is scored on a 5-point Likert-scale ranging from 0 to 4 . A total score above 33 indicates probable PTSD. The PCL-5 has demonstrated acceptable reliability and validity in previous studies (see Bovin et al., 2016). In the current sample, the reliability was high for the total scale, Cronbach's $\alpha=.97$, and the subscales, namely, reexperiencing Cronbach's $\alpha=.94$; avoidance, Cronbach's $\alpha$ $=.91$; negative alterations in cognition and mood (NACM), Cronbach's $\alpha=.94$; and arousal, Cronbach's $\alpha=.91$. In the present study, the total PCL-5 score was used.

\section{Data Analysis}

Analyses were conducted in three linked phases. First, a multivariate logistic regression was specified and estimated, with binary variables representing probable diagnostic status for anxiety, depression, and PTSD as dependent variables; and age, gender, and total number of childhood and adulthood trauma types as predictors. Second, an LCA that included gender as a 
covariate was conducted to determine the number of victimization classes. Latent class analysis a statistical method used to identify homogeneous groups or classes from categorical multivariate data. It is used to assess and summarize unobservable patterns in data that influence the relation between predictor and criterion-variables beyond observable quantitative variation. This is done by estimating one to six latent classes of respondents that differ qualitatively in their response patterns on predictor variables. In the present study, LCA was employed to determine the number and nature of patterns in response to the nine victimization variables. The fit of six models (i.e., one-class through six-class models) was assessed. The models were estimated using robust maximum likelihood (Yuan \& Bentler, 1997). The relative fit of the models was compared by using three information theory-based fit statistics, namely, the Akaike information criterion (AIC), the Bayesian information criterion (BIC), and the sample size-adjusted BIC (ssaBIC; Nylund et al., 2007). The model that produces the lowest values can be judged as the best model, provided that classes are meaningful and interpretable. In addition, the Lo-MendellRubin adjusted likelihood ratio test (LRT) was used to compare models with increasing numbers of latent classes. When a nonsignificant value (i.e., $p>.05$ ) occurs, this suggests that the model might not fit the data better than the previous model. The entropy of the latent class models was examined, with values closer to 1 suggestive of better group classification (Nylund et al., 2007).

Finally, using the three-step latent class approach, the relation between class membership and total scores on measures of anxiety, depression, and posttraumatic stress symptoms was computed using the DE3STEP command (Asparouhov \& Muthén, 2014). This approach estimates the relation between class membership and outcomes, while taking into account the imperfection of classification as indicated by the entropy values. The DE3STEP procedure is analogous to a multivariate analysis of data (MANOVA), where class membership is the independent variable and total scores are the dependent variables. The AUXILIARY command was used to conduct a logistic regression analysis to determine the likelihood of a probable 
diagnosis of PTSD, anxiety, and depression, based on class membership using the auxiliary DCAT command. All analyses were conducted using Mplus 8.1, and there were no missing data. A Kolmogorov-Smirnoff test indicated that data were nonnormally distributed on all outcome variables: depression, $D(1,051)=0.147, p<.001$; anxiety, $D(1,051)=0.161, p<.001$; PTSD, $D(1,051)=0.175, p<.001$; a visual examination of indicated that data were rightskewed. Robust maximum likelihood estimation produces correct point estimates, standard errors, and test statistics under conditions of nonnormality (Finney \& DiStefano, 2006).

\section{Results}

The frequencies of victimization experiences are presented in Table 1. Approximately two-thirds of the male participants $(68.7 \%)$ and $73.4 \%$ of the female participants had experienced at least one interpersonal trauma throughout their life. The most frequently reported childhood trauma was neglect, and the most frequently reported adulthood trauma was physical assault. Female participants were significantly more likely to report neglect and sexual abuse in childhood, and male participants were significantly more likely to report assault with weapon in childhood. In adulthood, male participants were significantly more likely to report physical assault and assault with weapon, and female participants were significantly more likely to report sexual assault. The mean scores on measures of PTSD, anxiety, and depression for the total sample were $19.02(S D=20.31), 13.41(S D=6.47)$, and $17.02(S D=7.65)$, respectively. Table 2 displays the results from the multivariate logistic regression predicting probable diagnostic status on anxiety, depression, and PTSD, predicted by age, gender, and total number of trauma types across childhood and adulthood. Younger age and higher levels of childhood and adult trauma exposure were all significantly associated with an increased risk of PTSD, anxiety, and depression. Being female was associated with an increased risk of depression.

The fit statistics for the LCA are presented in Table 3. Simulation studies suggest that the BIC is the best indicator of goodness-of-fit, signifying that Model 5 had the best fit for the data 
in the current study (Nylund et al., 2007). The LRT statistics and ssaBIC indicated that the sixclass solution was statistically superior to the five-class solution, but upon inspection of the 6class solution, the additional class resembled the pattern observed in an existing class and differed only quantitatively in the probability of endorsing the individual trauma types; therefore, this additional class did not satisfy the criterion of qualitative difference (Debowska, Willmott, Boduszek \& Jones, 2017). Taken together, the five-class solution was judged to be the bestfitting model. The average posterior probabilities ranged from .769 (Class 3) to .892 (Class 1). The profile plot and probabilities for the five-class solution are shown in Figure 1.

Class $1(n=87,8.3 \%)$ was characterized by the highest probabilities of all victimization types both during childhood and adulthood, apart from emotional abuse in childhood. This class encompassed $69.5 \%$ women $(n=57)$, had a mean participant age of 41.3 years $(S D=13.6)$, and was labelled the "lifetime polyvictimization" (POL). Class $2(n=144,13.7 \%)$ was characterized by elevated reports of sexual assault in both child and adulthood. This class comprised $96.5 \%$ ( $n$ $=139)$ women, had a mean participant age of 44.6 years $(S D=13.9)$, and was labelled the "sexual revictimization: (SV). Class $3(n=131,12.5 \%)$ was characterized by elevated reports of physical assault in both child and adulthood and was labelled the "physical revictimization" (PV) class. It comprised primarily men $(n=129,98.5 \%)$, with a mean age of 42.8 years $(S D=13.8)$. Class $4(n=272,25.9 \%)$ was characterized by elevated probabilities of emotional abuse, neglect, physical, and sexual abuse in childhood compared to the other classes, except for the POL class. This class was composed of $85.6 \%$ women $(n=230)$, with a mean participant age of 42.8 years $(S D=13.8)$, and was labelled the "childhood trauma" (CT) class. Class $5(n=417,39.7 \%)$ was the largest class and was characterized by the lowest probabilities of any form of victimization. This class comprised $40.3 \%$ women $(n=290)$, had a mean participant age of 50.4 years ( $S D$ =15.5), and was labelled the "limited victimization" (LV) class. Male and female participants were equally represented in the $\mathrm{LV}, \chi_{2}^{2}(1, N=1051)=0.33, p=.567$, and POL classes, $\chi_{2}^{2}(1, N=$ 
$1051)=0.24, p=.627$; whereas there were significantly more male participants in the PV class, $\chi_{2}(1, N=1051)=299.28, p<.001$, and significantly more female participants in the SV, $\chi_{2}(1, N$ $=1051)=59.54, p<.001$, and CT classes, $\left.\chi_{2}(1, N=1051)=43.28, p<.001\right)$. There was a significant difference in the average age of the observed groups: A one-way ANOVA, using a Welch-correction, identified two homogenous subsets, consisting of LV and PV classes and the POL, SV, and CT classes, respectively, $F_{\mathrm{Welch}}(4,342.5)=22.12, p<.001$.

Table 4 shows the mean scores and standard deviations for measures of depression, anxiety, and PTSD symptoms in each victimization class. Overall, the LV class reported the lowest severity of all clinical outcomes. Furthermore, participants in the LV class differed significantly from all other groups in their reporting of symptoms, with the exception of the PV class on measures of depression. The POL class and CT classes reported the highest and secondhighest mean scores, respectively, on all clinical measures and differed significantly from all other latent classes, including each other. The mean scores reported by participants in the PV and SV classes did not differ significantly from each other on depression, but the SV class reported significantly higher scores on measures of anxiety and PTSD compared to the PV class.

Finally, Table 4 also displays the results of the auxiliary logistic regression that testes the relation between class membership and probable diagnostic status. Overall, $24.6 \%$ of participants meet the criteria for probable PTSD, using a cutoff score of 33 on the PCL-5 as recommended by Weathers, Blake, et al (2013); 81.4\% met the criteria for depression, using a cutoff score of 10 on the PHQ-9 (Kroenke et al., 2001); and 61.1\% met the criteria for anxiety, using a cutoff score of 10 on the GAD-7 (Spitzer et al., 2006). The LV class was used as a reference class for comparison. Overall, the results shown in Table 4 were replicated in that the LV class had the lowest percentage of participants who endorsed a probable diagnosis of any disorder and differed significantly from all other classes. Likewise, the POL class reported the highest risk of endorsing any disorder and differed significantly from all other classes. Notably, the CT class 
reported the second-highest risk for endorsing any diagnosis and differed significantly from all other classes on PTSD, from all other classes apart from the SV class on anxiety, and only from the LV class on depression. Any differences in odds ratios for the PV and SV classes were statistically nonsignificant.

\section{Discussion}

In the present study, we aimed to examine patterns of lifetime interpersonal victimization based on exposure to childhood interpersonal trauma and adulthood physical and sexual victimization and assault with a weapon in a nationally representative panel of trauma-exposed adults from the United Kingdom. Furthermore, the current study examined whether individuals with different patterns of victimization differed in symptoms of anxiety, depression, and posttraumatic stress. The findings supported our first hypothesis (i.e., there would be varying patterns of lifetime interpersonal victimization, with at least one class categorized by exposure to interpersonal victimization during the developmental stages of childhood and adulthood), as three revictimization classes- $\mathrm{POL}, \mathrm{PV}$, and $\mathrm{SV}$ - emerged based on the exposure to multiple victimization types across the developmental stages of childhood and adulthood (Charak et al., 2018; Miron \& Orcutt, 2014). Additionally, there was a class of individuals who predominantly reported exposure to childhood interpersonal trauma and another class with low levels of victimization. The findings also indicated that male participants were more likely to be in the class characterized by physical revictimization, and female participants were more likely to be in the classes characterized by sexual revictimization and childhood trauma classes. In line with the traumagenic dynamics model and the cumulative trauma framework, we found that symptoms of anxiety, depression, and posttraumatic stress were more severe among individuals who had been exposed to lifetime revictimization (i.e., POL class) with multiple types of victimization (i.e., physical and sexual) during childhood and adulthood. Notably, the other two revictimization classes in which participants demonstrated higher probabilities of homotypic continuity of 
victimization - the SV and PV classes - were characterized by lower scores on measures of anxiety, depression, and posttraumatic stress symptoms when compared to the CT class; this lends partial support to our second hypothesis, which was that participants with membership in the latent classes characterized by revictimization experiences would report higher scores on measures of depression, anxiety, and posttraumatic stress symptoms as compared to those in other classes characterized by fewer victimization experiences or those that occurred during only one developmental phase. Overall, participants in all latent classes with substantial victimization experiences-POL, CT, PV, and SV—reported higher scores on measures of anxiety, depression, and posttraumatic stress symptoms compared to the LV class.

The present finding of a POL class comprising multiple types of childhood maltreatment histories and adulthood physical and sexual victimization is consistent with the literature indicating that exposure to more types of childhood traumatic experiences increases the likelihood of adulthood revictimization (Charak et al., 2019a; Classen et al., 2005; Widom et al., 2008). The presence of SV and PV classes attests to the presence of homotypic continuity of CSA and childhood physical abuse into adulthood sexual revictimization and physical revictimization, respectively. Although prior studies have demonstrated these findings, they have done so when examining the effect of childhood maltreatment types separately rather than simultaneously, as in the present study, for physical and sexual revictimization (Kimerling et al., 2007; Widom et al., 2008). The present findings suggest that even within revictimization experiences, there are different patterns, ranging from homotypic continuity to higher probabilities of type-specific revictimization (i.e., CSA into adulthood sexual assault; childhood physical abuse into adulthood physical assault) to exposure to multiple childhood interpersonal trauma along with an increased probability of exposure to different types of adulthood interpersonal trauma. 
Participants in the CT class demonstrated higher probabilities of childhood interpersonal trauma types compared to the PV and SV classes, whereas the latter two classes demonstrated higher probabilities of adult physical and sexual assault, respectively, compared to the CT class. This finding is in line with two recent systematic reviews that examined latent classes of lifetime traumatic events and indicated the presence of specific trauma classes of revictimization as well as latent classes characterized by exposure to childhood trauma (Contractor et al., 2018; O'Donnell et al., 2017). Additionally, the results of the present study identified a class with low probabilities of exposure to victimization; however, a separate class comprising mainly adulthood assault did not arise. The latter finding stands in contrast to previous literature that suggests the presence of experiences of sexual revictimization, childhood trauma, and adulthood assault among individuals with exposure to victimization across developmental stages (Charak et al., 2018; Walsh et al., 2012). Replication of current findings is warranted, and future studies focusing on samples from the United Kingdom should continue to explore classes of lifetime interpersonal victimization.

The present findings also demonstrate that individuals in the POL class had higher symptom scores and were more likely to score above the cutoff for a probable diagnosis of mental health problems, namely, depression, anxiety, and PTSD, than those in the other four classes. These findings support the results of prior studies indicating that individuals with revictimization experiences (Classen et al., 2005; Walsh et al., 2012) report more psychological problems. However, it was the CT class, characterized by exposure to predominantly childhood trauma types, that emerged as the class with the next-highest risk for mental health problems compared to the PV and SV revictimization classes and the LV class. Taken together, these findings suggest that early experiences of interpersonal trauma may disrupt the development of adaptive coping strategies (Cicchetti, Ganiban, \& Barnett, 1991), thus increasing the risk of mental health problems in adulthood. 
Participants in the SV class scored higher than those in the PV class on measures of anxiety and posttraumatic stress symptoms. This adds to the extant literature that suggests sexual revictimization experiences may lead to psychological sequelae of higher intensities. Although it may be that exposure to CSA, a precursor for revictimization, is more likely to elicit feelings of betrayal and mistrust of others at a younger age, which can exacerbate mental health problems (Gobin \& Freyd, 2009), it is important to be mindful that the severity of abuse and assault (e.g., the nature of the act, number of perpetrators, age of onset) also plays an important role in determining mental health problems (Charak et al., 2018). Future studies should account for the various characteristics that increase the severity of abuse and assault during childhood and adulthood.

The present findings also indicated a higher likelihood of receiving a PTSD diagnosis among participants in the POL class, followed by the CT, SV, and PV classes. Notably, $8.9 \%$ of participants in the LV class had a probable PTSD diagnosis, which is similar to the $8.3 \%$ reported by a national sample of U.S. adults (Kilpatrick et al., 2013). The percentage of participants with a PTSD diagnosis was slightly higher in the SV class compared to the PV class ( $20.8 \%$ vs. $17.6 \%$, respectively), although these findings were not significantly different. The current findings add to the literature that primarily focuses on sexual revictimization in women and states that it is exposure to multiple types of victimization across childhood and adulthood (i.e., POL) that is associated with more severe posttraumatic stress symptoms. Whether the higher scores on measures of depression, anxiety and posttraumatic stress are a long-term consequence of childhood maltreatment and, subsequently, a risk factor for revictimization (i.e., psychological vulnerabilities model; Culatta et al., 2017) or are a sequelae of revictimization (i.e., cumulative trauma model; O'Donnell et al., 2017), or both, can only be answered with the help of longitudinal research that can parse the risk factors from the consequences.

The present findings should be interpreted with the following limitations in mind. First, 
the present study relied on self-report measures to assess interpersonal victimization and related outcomes; this can lead to response bias as a result of an individual's ability to accurately recall the incident and/or their willingness to report experiences. Notably, prior studies have suggested that when behaviorally specific questions are used to assess victimization, as was the case in the present study, the accuracy of reporting increases rather than decreases (Fricker, Smith, Davis, \& Hanson, 2003). Additionally, some variables used to measure interpersonal trauma type (e.g., physical assault) were assessed using a single item, and this may have underestimated the prevalence of that trauma type. Second, data were collected through an online forum and pertained to individuals currently residing in the United Kingdom who reported exposure to a lifetime traumatic event. This may limit the generalization of findings to other samples. However, the use of online data collection forums may increase the likelihood individuals will report sensitive information in the absence of an interviewer (Tourangeau \& Smith, 1996) for the sample under study. Third, the cross-sectional design limited conclusions regarding the temporal relation among variables gauging revictimization and symptoms of depression, anxiety, and posttraumatic stress. Fourth, LCA is a data-driven analysis and, hence, has limited generalizations. Fifth, the present study did not examine the different patterns of victimization across gender and age, as the rates of certain types of victimization were very low across ages and for both female and male participants; for example, assault with a weapon was reported by $4.2 \%$ and $8.7 \%$, respectively. In such cases, the application of mixture models becomes difficult, as the expectation is to find more than one class, and simulation studies suggest that latent classes that contain less than 5\% of the sample cause problems with model convergence (Nylund et al., 2007). Nonetheless, future studies should attempt to investigate the different patterns of victimization across gender and age or developmental stages. Sixth, the present study did not take into consideration other characteristics of childhood or adulthood assault, such as age of onset (Kaplow \& Widom, 2007) and the nature of the acts (Charak et al., 2018), which t have 
important implications regarding the psychological consequences associated with victimization across the lifespan. This is important as previous research has identified that there are many different forms of sexual abuse that can co-occur (Shevlin, Murphy, Elklit, Murphy, \& Hyland, 2018) and have complex associations with adult psychopathology (McElroy et al., 2016). Finally, no information on race or ethnicity was collected.

Nonetheless, the present findings add to the extant literature by going beyond the assessment of sexual revictimization and finding patterns of victimization reflective of latent classes of revictimization, childhood trauma, and limited exposure to victimization in a large sample of trauma-exposed individuals from the United Kingdom. Although all forms of abuse and assault can be potentially harmful, recognizing the heterogeneity in victimization experiences, in childhood and adulthood, may inform clinical interventions, as different types of victimization may be differentially associated with negative outcomes. For example, individuals with higher probabilities of childhood interpersonal trauma types (i.e., those in the POL and CT classes) were at a higher risk of depression, anxiety, and PTSD than those in the other classes. In such instances, cognitive behavioral therapies (CBT), such as trauma-focused CBT, have been found to effectively reduce symptoms of PTSD, depression, and anxiety in children and youth who report traumatic experiences (Cohen, Mannarino, \& Deblinger, 2012). Adult survivors of revictimization experiences may benefit from other CBT-based therapies, such as prolonged exposure and cognitive processing therapy, to help create a new understanding and conceptualization of the traumatic event in order to alleviate PTSD, depression, anxiety, and guilt (Foa, Rothbaum, Riggs, \& Murdock, 1991; Resick, Williams, Sovak, Monson, \& Gradus, 2012). Although histories of trauma and victimization are a part of clinical assessment for PTSD, the present findings suggest that mental health care providers and clinicians should routinely inquire about trauma history from clients seeking treatment for other mental health problems, including depression and anxiety. Early identification of traumatic events in children is important 
and calls for educational programs directed at creating awareness regarding screening and assessment of childhood trauma among medical and other health care professionals. 


\section{References}

Armour, C., \& Sleath, E. (2014). Assessing the co-occurrence of intimate partner violence domains across the life-course: Relating typologies to mental health. European Journal of Psychotraumatology, 5. https://doi.org/10.3402/ejpt.v5.24620

Asparouhov, T., \& Muthén, B. (2014). Auxiliary variables in mixture modeling: Three-step approaches using Mplus. Structural Equation Modeling: A Multidisciplinary Journal, 21, 1-13. https://doi.org/10.1080/10705511.2014.915181

Ballard, E. D., Van Eck, K., Musci, R. J., Hart, S. R., Storr, C. L., Breslau, N., \& Wilcox, H. C. (2015). Latent classes of childhood trauma exposure predict the development of behavioral health outcomes in adolescence and young adulthood. Psychological Medicine, 45(15), 3305-3316. https://doi.org/10.1017/S0033291715001300

Black, M. C., Basile, K. C., Smith, S. G., Walters, M. L., Merrick, M. T., Chen, J., \& Stevens, M. R. (2010). National Intimate Partner and Sexual Violence Survey 2010 summary report. Atlanta, GA: National Center for Injury Prevention and Control, Centers for Disease Control and Prevention. Retrieved from https://doi.org/10.1093/oxfordhb/9780199844654.013.0003

Bovin, M. J., Marx, B. P., Weathers, F. W., Gallagher, M. W., Rodriguez, P., Schnurr, P. P., \& Keane, T. M. (2016). Psychometric properties of the PTSD Checklist for Diagnostic and Statistical Manual of Mental Disorders (5th edition; PCL-5) in veterans. Psychological Assessment, 28, 1379-1391. http://doi.org/10.1037/pas0000254

Cicchetti, D., Ganiban, J., \& Barnett, D. (1991). Contributions from the study of high-risk populations to understanding the development of emotion regulation. In J. Garber \& K. A. Dodge (Eds.), The development of emotion regulation and dysregulation (pp. 15-48). Cambridge, UK: Cambridge University Press. 
Contractor, A. A., Caldas, S., Fletcher, S., Shea, M. T., \& Armour, C. (2018). Empirically derived lifespan polytraumatization typologies: A systematic review. Journal of Clinical Psychology, 74, 1137-1159. https://doi.org/10.1002/jclp.22586

Charak, R., Byllesby, B. M., Roley, M. E., Claycomb, M. A., Durham, T. A., Ross, J., ... Elhai, J. D. (2016). Latent classes of childhood poly-victimization and associations with suicidal behavior among adult trauma victims: Moderating role of anger. Child Abuse and Neglect, 62, 19-28. https://doi.org/10.1016/j.chiabu.2016.10.010

Charak, R., DiLillo, D., Messman-Moore, T. L., \& Gratz, K. L. (2018). Latent classes of lifetime sexual victimization characteristics in women in emerging adulthood: Differential relations with emotion dysregulation. Psychology of Violence, 8, 570-579. https://doi.org/10.1037/vio0000154

Charak, R., Eshelman, L. R., \& Messman-Moore, T. L. (2019a). Latent classes of childhood maltreatment, adult sexual assault, and revictimization in men: Difficulties in masculinity, anger, and substance use. Psychology of Men and Masculinity, 20, 503-514. https://doi.org/10.1037/men0000185

Charak, R., Ford, J. D., Modrowski, C. A., \& Kerig, P. K. (2019). Polyvictimization, emotion dysregulation, symptoms of posttraumatic stress disorder, and behavioral health problems among justice-involved youth: A latent class analysis. Journal of Abnormal Child Psychology, 47, 287-298. https://doi.org/10.1007/s10802-018-0431-9

Charak, R., \& Koot, H. M. (2015). Severity of maltreatment and personality pathology in adolescents of Jammu, India: A latent class approach. Child Abuse and Neglect, 50, 5666. https://doi.org/10.1016/j.chiabu.2015.05.010

Charak, R., Koot, H. M., Dvorak, R. D., Elklit, A., \& Elhai, J. D. (2015). Unique versus cumulative effects of physical and sexual assault on patterns of adolescent substance use. Psychiatry Research, 230, 763-769. https://doi.org/10.1016/j.psychres.2015.11.014 
Classen, C. C., Palesh, O. G., \& Aggarwal, R. (2005). Sexual revictimization: A review of the empirical literature. Trauma, Violence, \& Abuse, 6, 103-129. https://doi.org/10.1177/1524838005275087

Cohen, J. A., Mannarino, A. P., \& Deblinger, E. (2012). Trauma-focused CBT for children and adolescents: treatment applications. New York: The Guildford Press.

Culatta, E., Clay-Warner, J., Boyle, K. M., \& Oshri, A. (2017). Sexual revictimization: A routine activity theory explanation. Journal of Interpersonal Violence. Advance online publication. https://doi.org/10.1177/0886260517704962

Debowska, A., Willmott, D., Boduszek, D., \& Jones, A. D. (2017). What do we know about child abuse and neglect patterns of co-occurrence? A systematic review of profiling studies and recommendations for future research. Child Abuse \& Neglect, 70, 100-111. https://doi.org/10.1016/j.chiabu.2017.06.014

Felitti, V. J., Anda, R. F., Nordenberg, D., Williamson, D. F., Spitz, A. M., Edwards, V., ... Marks, J. S. (1998) Relationship of childhood abuse and household dysfunction to many of the leading causes of death in adults: The adverse childhood experiences (ACE) study. American Journal of Preventive Medicine. 14, 245-258. https://doi.org/10.1016/j.amepre.2019.04.001

Filipas, H. H., \& Ullman, S. E. (2006). Child sexual abuse, coping responses, self-blame, posttrauamtic stress disorder, and adult sexual revictimization. Journal of Interpersonal Violence, 21, 652-672. https://doi.org/10.1177/0886260506286879

Finkelhor, D., \& Browne, A. (1985). The traumatic impact of child sexual abuse: A conceptualization. American Journal of Orthopsychiatry, 55, 530-541. http://doi.org/10.1111/j.1939-0025.1985.tb02703.x

Foa, E. B., Rothbaum, B. O., Riggs, D. S., \& Murdock, T. B. (1991). Treatment of posttraumatic stress disorder in rape victims: A comparison between cognitive-behvaioral procedures 
and counseling. Journal of Consulting and Clinical Psychology, 59, 715723. https://doi.org/10.1037/0022-006x.59.5.715

Ford, J. D., Charak, R., Modrowski, C. A., \& Kerig, P. K. (2018). PTSD and dissociation symptoms as mediators of the relationship between polyvictimization and psychosocial and behavioral problems among justice-involved adolescents. Journal of Trauma and Dissociation, 19, 325-346. https://doi.org/10.1080/15299732.2018.1441354

Fricker, A. E., Smith, D. W., Davis, J. L., \& Hanson, R. F. (2003). Effects of context and question type on endorsement of childhood sexual abuse. Journal of Traumatic Stress, 16, 265-268. https://doi.org/10.1023/A:1023748124626

Gobin, R., \& Freyd, J. J. (2009). Betrayal and revictimization: Preliminary findings. Psychological Trauma: Theory, Research, Practice, and Policy, 1, 242257. https://doi.org/10.1037/a0017469

Houston, J. E., Shevlin, M., Adamson, G., \& Murphy, J. (2011). A person-centred approach to modelling population experiences of trauma and mental illness. Social Psychiatry and Psychiatric Epidemiology, 46, 149-157. https://doi.org/10.1007/s00127-009-0176-4

Jankowski, M. K., Leitenberg, H., Henning, K., \& Coffey, P. (2002). Parental caring as a possible buffer against sexual revictimization in young adult survivors of child sexual abuse. Journal of Traumatic Stress, 15, 235-244. https://doi.org/10.1023/a:1015259412746

Kaplow, J. B., \& Widom, C. S. (2007). Age of onset of child maltreatment predicts long-term mental health outcomes. Journal of Abnormal Psychology, 116, 176-187. https://doi.org/10.1037/0021-843X.116.1.176

Kilpatrick, D., Resnick, H. S., Milanak, M. E., Miller, M. W., Keyes, K. M., \& Friedman, M. J. (2013). National estimates of exposure to traumatic events and PTSD prevalence using 
DSM-IV and DSM-5 criteria. Journal of Traumatic Stress, 26, 537-547. https://doi.org/10.1002/jts.21848

Kimerling, R., Alvarez, J., Pavao, J., Kaminski, A., \& Baumrind, N. (2007). Epidemiology and consequences of women's revictimization. Women's Health Issues, 17, 101-106. https://doi.org/10.1016/j.whi.2006.12.002

Kroenke, K., Spitzer, R., \& Williams, W. (2001). The PHQ-9: Validity of a brief depression severity measure. Journal of General Internal Medicine, 16, 606-616. https://doi.org/10.1046/j.1525-1497.2001.016009606.x

Lagdon, S., Armour, C., \& Stringer, M. (2014). Adult experience of mental health outcomes as a result of intimate partner violence victimisation: A systematic review. European Journal of Psychotraumatology, 5, 1-12. https://doi.org/10.3402/ejpt.v5.24794

McElroy, E., Shevlin, M., Elklit, A., Hyland, P., Murphy, S., \& Murphy, J. (2016). Prevalence and predictors of Axis I disorders in a large sample of treatment-seeking victims of sexual abuse and incest. European Journal of Psychotramatology, 7, 30686. https://doi.org/10.3402/ejpt.v.7.30686

McLafferty, M., Armour, C., McKenna, A., O’Neill, S., Murphy, S., \& Bunting, B. (2015). Childhood adversity profiles and adult psychopathology in a representative Northern Ireland study. Journal of Anxiety Disorders, 35, 42-48. https://doi.org/10.1016/j.janxdis.2015.07.004

Messman-Moore, T. L., \& Long, P. J. (2003). The role of childhood sexual abuse sequelae in the sexual revictimization of women: An empirical review and theoretical reformulation. Clinical Psychology Review, 23, 537-571. https://doi.org/10.1016/s0272-7358(02)002039

Miron, L. R., \& Orcutt, H. K. (2014). Pathways from childhood abuse to prospective revictimization: Depression, sex to reduce negative affect, and forecasted sexual 
behavior. Child Abuse and Neglect, 38, 1848-1859.

https://doi.org/10.1016/j.chiabu.2014.10.004

Nylund, K.L., Asparouhov, T., \& Muthén, B.O. (2007). Deciding on the number of classes in latent class analysis and growth mixture modeling: A Monte Carlo simulation study. Structural Equation Modeling, 14, 535-569. https://doi.org/10.1080/10705510701575396

O’Donnell, M. L., Schaefer, I., Varker, T., Kartal, D., Forbes, D., Bryant, R. A. A., ... Steel, Z. (2017). A systematic review of person-centered approaches to investigating patterns of trauma exposure. Clinical Psychology Review, 57, 208-225. https://doi.org/10.1016/j.cpr.2017.08.009

Resick, P. A., Williams, L. F., Suvak, M. K., Monson, C. M., \& Gradus, J. L. (2012). Long-term outcomes of cognitive-behvaioral treatments for posttraumatic stress disorder among female rape survivors. Journal of Clinical and Consulting Psychology, 80, 201-210. https://doi.org/10.1037/a0026602

Rivera, P. M., Fincham, F. D., \& Bray, B. C. (2018). Latent classes of maltreatment: A systematic review and critique. Child Maltreatment, 23, 3-24. https://doi.org/10.1177/1077559517728125

Shevlin, M., Murphy, S., Elklit, A., Murphy, J., \& Hyland, P. (2018). Typologies of child sexual abuse: An analysis of multiple abuse acts among a large sample of Danish treatmentseeking survivors of childhood sexual abuse. Psychological Trauma, 10, 263-269. https://doi.org/10.1037/tra0000268

Spitzer, R. L., Kroenke, K., Williams, J. B., \& Löwe, B. (2006). A brief measure for assessing generalized anxiety disorder: The GAD-7. Archives of Internal Medicine, 166, 10921097. https://doi.org/10.1001/archinte.166.10.1092 
Tourangeau, R., \& Smith, T. W. (1996). Asking sensitive questions: The impact of data collection mode, question format, and question context. Public Opinion Quarterly, 60, 275-304. https://doi.org/10.1086/297751

Ullman, S. E., Najdowski, C. J., \& Filipas, H. H. (2009). Child sexual abuse, post-traumatic stress disorder, and substance use: Predictors of revictimization in adult sexual assault survivors. Journal of Child Sexual Abuse, 18, 367-385. https://doi.org/10.1080/10538710903035263

Walsh, K., Danielson, C. K., McCauley, J. L., Saunders, B. E., Kilpatrick, D. G., \& Resnick, H. S. (2012). National prevalence of posttraumatic stress disorder among sexually revictimized adolescent, college, and adult household-residing women. Archives of General Psychiatry, 69, 935-942. https://doi.org/10.1001/archgenpsychiatry.2012.132

Weathers, F. W., Blake, D. D., Schnurr, P. P., Kaloupek, D. G., Marx, B. P., \& Keane, T. M. (2013). The life events checklist for DSM-5 (LEC-5). Instrument available from the National Center for PTSD at http://www.ptsd.va.gov

Weathers, F. W., Litz, B. T., Keane, T. M., Palmieri, P. A., Marx, B. P., \& Schnurr, P. P. (2013). The PTSD Checklist for DSM-5 (PCL-5). Scale available from the National Center for PTSD at http://www.ptsd.va.gov

Widom, C. S., Czaja, S. J., \& Dutton, M. A. (2008). Childhood victimization and lifetime revictimization. Child Abuse and Neglect, 32, 785-796. https://doi.org/10.1016/j.chiabu.2007.12.006

Yuan, K. H., \& Bentler, P. M. (1997). Mean and covariance structural analysis: Theoretical and practical improvements. Journal of the American Statistical Association, 92, 767-774. https://doi.org/10.1080/01621459.1997.10474029 
Table 1

Frequency of Interpersonal Trauma Types in a United Kingdom-Based Community Sample

\begin{tabular}{|c|c|c|c|c|c|c|c|c|}
\hline \multirow{2}{*}{$\begin{array}{l}\text { Trauma type } \\
\text { During childhood }\end{array}$} & \multicolumn{2}{|c|}{$\begin{array}{c}\text { Total } \\
(N=1,051)\end{array}$} & \multicolumn{2}{|c|}{$\begin{array}{c}\text { Men } \\
(n=332)\end{array}$} & \multicolumn{2}{|c|}{$\begin{array}{l}\text { Women } \\
(n=719)\end{array}$} & \multirow[t]{2}{*}{$\chi_{2}^{2}(1, N=1,051)$} & \multirow[t]{2}{*}{$p$} \\
\hline & $n$ & $\%$ & $n$ & $\%$ & $n$ & $\%$ & & \\
\hline Emotional abuse & 380 & 36.2 & 107 & 32.2 & 273 & 38.0 & 3.24 & .083 \\
\hline Emotional or physical & 391 & 37.2 & 102 & 30.7 & 289 & 40.2 & 8.32 & .004 \\
\hline \multicolumn{9}{|l|}{ neglect } \\
\hline Witnessing domestic & 164 & 15.6 & 46 & 13.9 & 118 & 16.4 & 0.94 & .332 \\
\hline \multicolumn{9}{|l|}{ violence } \\
\hline Physical assault & 340 & 32.4 & 111 & 33.4 & 229 & 31.8 & 0.19 & .660 \\
\hline Assault with a weapon & 59 & 5.6 & 29 & 8.7 & 30 & 4.2 & 8.08 & .004 \\
\hline Sexual abuse/assault & 287 & 27.3 & 63 & 19.0 & 224 & 31.2 & 16.36 & .001 \\
\hline \multicolumn{9}{|l|}{ During adulthood } \\
\hline Physical assault & 295 & 28.1 & 110 & 33.1 & 185 & 25.7 & 5.80 & .016 \\
\hline Assault with a weapon & 94 & 8.9 & 45 & 13.6 & 49 & 6.8 & 11.85 & .001 \\
\hline
\end{tabular}


Table 2

Odds Ratios (ORs) From the Multivariate Logistic Regression for Age, Gender, and Total Number of Trauma Exposures

\begin{tabular}{|c|c|c|c|c|c|c|}
\hline \multirow[t]{2}{*}{ Variable } & \multicolumn{2}{|c|}{ PTSD } & \multicolumn{2}{|c|}{ Anxiety } & \multicolumn{2}{|c|}{ Depression } \\
\hline & $O R$ & $S E$ & $O R$ & $S E$ & OR & $S E$ \\
\hline Age & 0.964 & $0.006 * * *$ & 0.970 & $0.005 * * *$ & 0.982 & $0.006^{* * *}$ \\
\hline Gender & 1.227 & 0.237 & 1.320 & 0.201 & 1.492 & $0.269 *$ \\
\hline Total childhood trauma & 1.484 & $0.143^{* * *}$ & 1.277 & $0.123 *$ & 1.361 & $0.179 * * *$ \\
\hline Total adulthood trauma & 1.593 & $0.082 * * *$ & 1.434 & $0.076 * * *$ & 1.517 & $0.116 *$ \\
\hline
\end{tabular}

Note. PTSD = posttraumatic stress disorder.

$* p<.05 . * * p<.01 . * * * p<.001$. 
Table 3

Latent Class Analysis Fit Statistics for Interpersonal Child and Adult Victimization, With

Gender as a Covariate

\begin{tabular}{lccccccc}
\hline Number of & Log & AIC & BIC & ssaBIC & LRT & LRT & Entropy \\
Classes & likelihood & & & & & \\
& & & & & & & \\
\hline 2 & $-4,335.583$ & $8,711.166$ & $8,810.316$ & $8,746.793$ & $1,052.755$ & $<$ & .774 \\
& & & & & .001 & \\
3 & $-4,247.498$ & $8,556.996$ & $8,710.678$ & $8,612.217$ & 173.898 & .006 & .783 \\
4 & $-4,194.100$ & $8,472.201$ & $8,680.416$ & $8,547.017$ & 105.417 & .031 & .747 \\
5 & $-4,144.222$ & $8,394.443$ & $8,657.191$ & $8,488.855$ & 98.471 & .109 & .717 \\
6 & $-4,119.708$ & $8,367.416$ & $8,684.696$ & $8,481.422$ & 48.395 & .024 & .729
\end{tabular}

Note. $N=1,051$. AIC $=$ Akaike information criteria; $\mathrm{BIC}=$ Bayesian information criteria;

ssaBIC $=$ sample-size adjusted BIC $;$ LRT $=$ Lo-Mendell-Rubin adjusted likelihood ratio test. 
Table 4

Mean Scores and Standard Errors on Measures of Clinical Disorder Symptoms, and Odds

Ratios, 95\% Confidence Intervals, and Percentage of Participants Who Qualified for a Probable

Diagnosis of Posttraumatic Stress Disorder (PTSD), Anxiety, and Depression, Compared to the

Limited Victimization Class (Reference Group)

\begin{tabular}{|c|c|c|c|c|c|}
\hline & \multicolumn{2}{|c|}{ Symptoms } & \multicolumn{3}{|c|}{ Probable Diagnosis } \\
\hline & $M$ & $S E$ & $O R$ & $95 \% \mathrm{CI}$ & $\%$ \\
\hline \multicolumn{6}{|c|}{ POL classe } \\
\hline \multicolumn{6}{|c|}{$(n=87)$} \\
\hline Anxiety & 19.35abcd & 1.02 & 12.1 abcd & {$[5.36,27.36]$} & 86.2 \\
\hline Depression & 25.34abcd & 1.10 & 9.48abcd & {$[3.34,26.87]$} & 94.3 \\
\hline PTSD & 51.34abcd & 3.93 & $33.63 \mathrm{abcd}$ & {$[16.35,69.43]$} & 62.1 \\
\hline \multicolumn{6}{|c|}{ SV classc } \\
\hline \multicolumn{6}{|c|}{$(n=144)$} \\
\hline Anxiety & 13.28abde & 0.70 & $3.58 \mathrm{ae}$ & {$[1.99,6.44]$} & 68.8 \\
\hline Depression & 17.12ade & 0.89 & $4.54 \mathrm{ae}$ & {$[1.86,11.12]$} & 84.7 \\
\hline
\end{tabular}




\begin{tabular}{|c|c|c|c|c|c|}
\hline \multicolumn{5}{|c|}{ INTERPERSONAL VICTIMIZATION AND MENTAL HEALTH } & \multirow{2}{*}{$\begin{array}{c}34 \\
20.8\end{array}$} \\
\hline PTSD & 16.04abde & 2.00 & 4.09ade & {$[1.80,9.32]$} & \\
\hline \multicolumn{6}{|c|}{ PV classb } \\
\hline \multicolumn{6}{|c|}{$(n=131)$} \\
\hline Anxiety & $11.3 \mathrm{cde}$ & 0.52 & 1.90ade & {$[1.00,3.47]$} & 49.6 \\
\hline Depression & 15.05 ade & 0.72 & 2.26ade & {$[1.07,4.75]$} & 78.6 \\
\hline PTSD & $11.01_{\mathrm{cde}}$ & 1.57 & 3.64ade & {$[1.47,9.04]$} & 17.6 \\
\hline \multicolumn{6}{|c|}{ CT classd } \\
\hline \multicolumn{6}{|c|}{$(n=272)$} \\
\hline Anxiety & 16.51 abce & 0.64 & $4.59 \mathrm{abe}$ & {$[3.06,6.90]$} & 76.1 \\
\hline Depression & $20.40 \mathrm{abce}$ & 0.75 & $6.13 \mathrm{a}$ & {$[3.39,11.09]$} & 91.2 \\
\hline PTSD & 27.39 abce & 2.41 & 10.64abce & {$[5.81,19.47]$} & 42.3 \\
\hline \multicolumn{6}{|c|}{ LV classa } \\
\hline \multicolumn{6}{|c|}{$(n=417)$} \\
\hline Anxiety & 10.73 cde & 0.28 & - & - & 47.0 \\
\hline Depression & 13.39 bcde & 0.32 & - & - & 71.9 \\
\hline PTSD & 9.43 cde & $(0.77)$ & - & - & 8.9 \\
\hline
\end{tabular}


Note. $\mathrm{POL}=$ lifetime polyvictimization $\mathrm{SV}=$ sexual revictimization $; \mathrm{PV}=$ physical

revictimization $; \mathrm{CT}=$ childhood trauma $\mathrm{LV}=$ limited victimization .

abcde Indicates the classes from which the respective class scores on clinical outcomes differ significantly. 


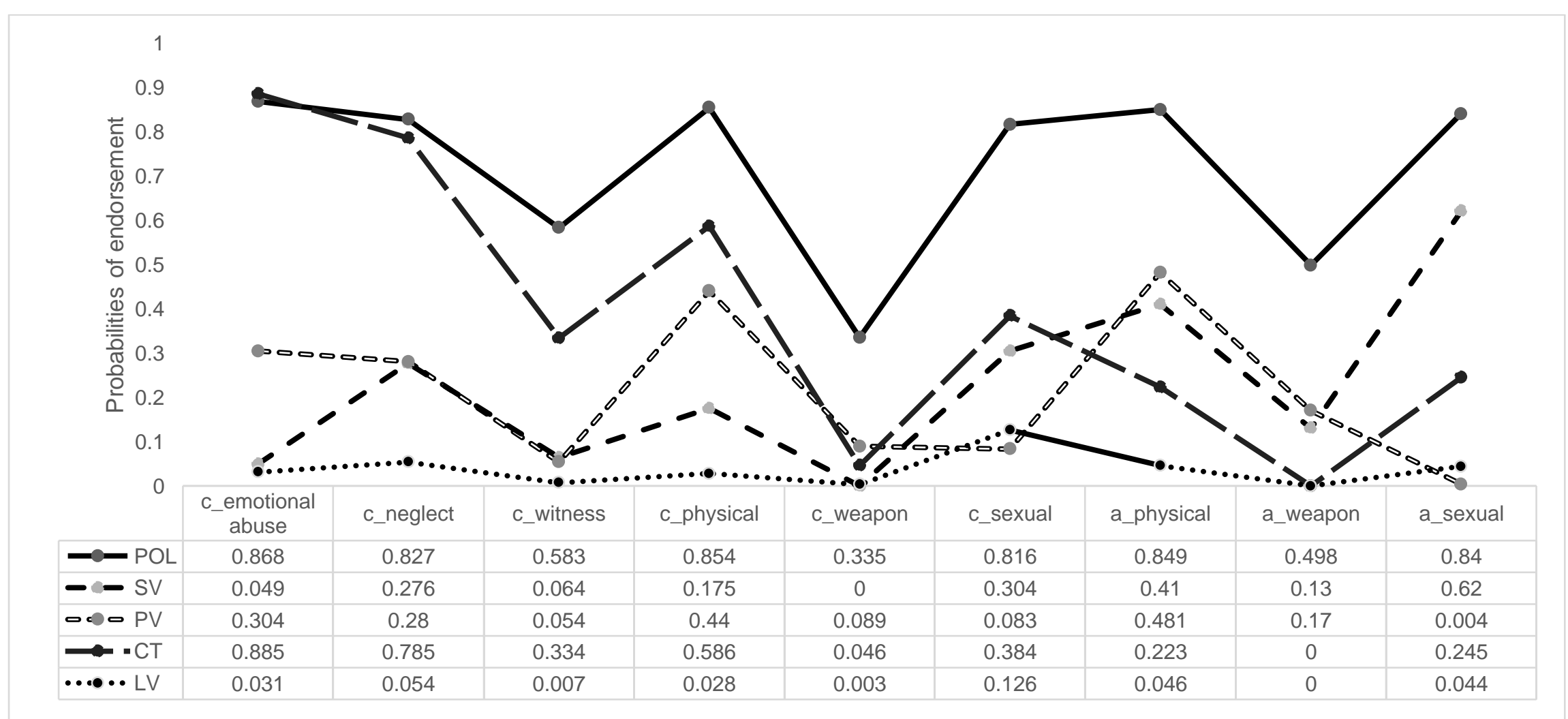

Figure 1. Profile plot and probabilities from the latent class analysis of victimization variables.

c_emotional abuse $=$ childhood emotional abuse $;$ c_neglect $=$ childhood neglect $;$ c_physical $=$ childhood physical abuse; c_weapon = threatened by a weapon during childhood; c_sexual = 
childhood sexual abuse; a_physical = adulthood physical assault; a_weapon = threatened by a weapon during adulthood; a_sexual = adulthood sexual assault; $\mathrm{POL}=$ lifetime

polyvictimization; $\mathrm{SV}=$ sexual revictimization; $\mathrm{PV}=$ physical revictimization $; \mathrm{CT}=$ childhood trauma; $\mathrm{LV}=$ limited victimization. 